\title{
Perioperative changes in pelvic tilt after hip resurfacing using a direct lateral approach
}

\author{
Linsen T. Samuel ${ }^{1}$, Jared Warren ${ }^{1}$, Jacob M. Rabin ${ }^{1}$, Alexander J. Acuña ${ }^{2}$, Amy Shuster ${ }^{3}$, Jordan Patterson ${ }^{3}$, \\ Michael A. Mont ${ }^{4}$, Peter J. Brooks ${ }^{1}$ \\ ${ }^{1}$ Department of Orthopaedic Surgery, Cleveland Clinic Foundation, Cleveland, OH, USA; ${ }^{2}$ Case Western Reserve University School of Medicine, \\ Cleveland, OH, USA; ${ }^{3}$ Ohio University Heritage College of Osteopathic Medicine, Athens, OH, USA; ${ }^{4}$ Department of Orthopedic Surgery, Lenox \\ Hill Hospital at Northwell Health, New York, NY, USA \\ Contributions: (I) Conception and design: LT Samuel, PJ Brooks; (II) Administrative support: LT Samuel, A Shuster, J Patterson; (III) Provision of \\ study materials or patients: PJ Brooks; (IV) Collection and assembly of data: LT Samuel, A Shuster, J Patterson; (V) Data analysis and interpretation: \\ LT Samuel, J Warren; (VI) Manuscript writing: All authors; (VII) Final approval of manuscript: All authors. \\ Correspondence to: Michael A. Mont, MD. 100 East $77^{\text {th }}$ Street, New York, NY 10075, USA. Email: montm@ccf.org.
}

Background: Proper positioning of the acetabular component is critical for prevention of dislocation and excessive wear for total hip arthroplasty (THA) and hip resurfacing. Consideration of preoperative pelvic tilt (PT) may aid in acetabular component placement. The purpose of this study was to investigate how PT changes after hip resurfacing, via pre and post-operative radiographic analysis of anterior pelvic plane (APP), and whether radiographic analysis of the APP is a reproducible method for evaluating PT in resurfaced hips.

Methods: A consecutive group of 228 patients from a single surgeon who had hip resurfacing were evaluated. We obtained X-rays from an institutional database for these patients who had their surgeries between January $1^{\text {st }}, 2014$ to December $31^{\text {st }}$, 2016. PT was measured by two observers before and after resurfacing utilizing a standardized radiographic technique. Correlation coefficients were calculated for PT measurements between observers, and pre- and post-surgery.

Results: Mean preoperative PT was $0.7^{\circ}\left(\mathrm{SD} \pm 6.6^{\circ}\right)$ and $0.4^{\circ}\left(\mathrm{SD} \pm 6.1^{\circ}\right)$. Mean post-operative PT was $-1.2^{\circ}\left(\mathrm{SD} \pm 6.2^{\circ}\right)$ and $-1.2^{\circ}\left(\mathrm{SD} \pm 6.0^{\circ}\right)$. Correlations between pre and post-operative PT were $\mathrm{R}=0.829$ $(\mathrm{P}<0.001)$ and $\mathrm{R}=0.837(\mathrm{P}<0.001) .80 .6 \%$ to $82.5 \%$ of patients had variation $<5^{\circ}, 15.8 \%$ to $17.8 \%$ had variation between $5-10^{\circ}$, and $1.6 \%$ to $1.8 \%$ had a variation $>10^{\circ}$. Intraclass correlation coefficients between observers were $\mathrm{R}=0.987$ (95\% CI, 0.963-0.981; $\mathrm{P}<0.001$ ) preoperatively, and $\mathrm{R}=0.985$ (95\% CI, 0.963-0.981; $\mathrm{P}<0.001)$ postoperatively.

Conclusions: After hip resurfacing arthroplasty (HRA), the mean difference between preoperative and postoperative PT was less than $1^{\circ}$. These results suggest that near-native PT is maintained with consistency after hip resurfacing, a finding that is variable following THA. Since variations in PT affect functional acetabular position, these results support the use of PT measurement in pre-operative planning for hip arthroplasty with a high degree of inter-observer reliability.

Keywords: Pelvic tilt (PT); anterior pelvic plane (APP) angle; hip resurfacing; perioperative changes; direct lateral

Submitted Apr 30, 2019. Accepted for publication May 23, 2019.

doi: $10.21037 /$ atm.2019.05.67

View this article at: http://dx.doi.org/10.21037/atm.2019.05.67 


\section{Introduction}

As an alternative to total hip arthroplasty (THA), hip resurfacing arthroplasty (HRA) is suited to younger, more active patients due to conservation of femoral bone, preservation of native biomechanical hip parameters, physiological femoral loading, and overall improved joint stability (1). As in THA, proper positioning of the acetabular component is critical for successful outcome in hip resurfacing, to ensure adequate range of motion (ROM), and to prevent dislocation and wear, two of the most prevalent causes of failed surgery (2-9). While dislocation is less common after resurfacing relative to THA, wear of metalon-metal resurfacing bearings may lead to metallosis, and pseudotumor formation, and can arise from increased edge loading due to malposition of the acetabular cup (8,10-13).

In both THA and hip resurfacing, cup placement has traditionally been guided by a "safe zone" of inclination and anteversion in reference to the anterior-pelvic-plane (APP), defined by the two anterior superior iliac spines (ASIS) and the pubic symphysis $(2,11,14-16)$. Additionally, many computer-navigated systems rely on CT-scan reconstruction of the APP to guide placement of the cup $(11,17,18)$. The APP has historically been assumed to be vertical in standing position (11,17-19). However, this is not the case due to substantial individual variation in functional orientation of the pelvis in the sagittal plane (20). The parameter that accounts for this variation, known as pelvic tilt (PT), can be defined as the angle between the APP and coronal plane, wherein standing position normal PT is indicated by APP aligned with the coronal plane, posterior PT by ASIS posterior to the pubic symphysis, and anterior PT by ASIS anterior to the pubic symphysis (see Figure 1).

Studies have shown that PT is a primary determinant of functional orientation of the acetabulum $(21,22)$. It was also demonstrated that it is a dynamic parameter that changes with position and activity (22). The degree of this change, in addition to static PT in functional positions, has been shown to vary substantially between patients $(2,14,18-20)$. There is increasing consensus that lack of consideration of this variation can result in suboptimal functional orientation of the acetabular component, especially when APP is relied on as the intraoperative anatomical reference to guide its positioning $(6-8,14,16,19,23)$. Evidence suggests that consideration of pre-operative $\mathrm{PT}$ in functional positions can improve outcomes after THA, particularly when quantified and incorporated into computer-navigation systems $(15,18,24)$. PT may also affect the functional position of the acetabular component after resurfacing, particularly in respect to anteversion, decreasing anteversion in case of anterior PT, and increasing anteversion in case of posterior PT. Patients with excessive posterior PT after surgery may therefore be at risk of increased edge-loading, wear, and associated complications.

Despite accuracy of cup placement adjusted for preoperative PT, this may be confounded by changes in PT caused by surgery $(7,8,18)$. Improved understanding of the effects of hip arthroplasty on native PT is therefore warranted. Although several studies have investigated changes in PT after THA $(4,17,20,25-29)$ (see Table 1), similar investigations have not been conducted in the context of hip resurfacing. The purpose of this study was to investigate how PT changes after hip resurfacing, via pre and post-operative radiographic analysis of anterior pelvic plane (APP), and whether radiographic analysis of the APP is a reproducible method for evaluating PT in resurfaced hips.

\section{Methods}

IRB approval was obtained for this study.

\section{Data collection and patient selection}

This retrospective study consisted of 228 patients who underwent hip resurfacing by a single surgeon between January $1^{\text {st }}, 2014$ and December $31^{\text {st }}, 2016$ at a large academic institution. All patients were male, had no previous hip implantations, and underwent a unilateral procedure with implantation of the Birmingham Hip Resurfacing (BHR) system. A direct lateral approach was used in all cases. PT was measured by two observers using patient radiographs with the electronic medical record, taken before, and 1 year after hip resurfacing. Radiographs were lateral views of the pelvis with patients in standing position. The observer drew two lines on digital images. One line, representing the APP, connected the ASIS to the pubic symphysis. If both ASIS were not superimposed in the lateral view, a line connecting the ASIS was drawn and the midpoint was used as the ASIS reference point, which was then connected to the pubic symphysis (see Figure 1). The second line, representing the vertical plane, intersected the first line at the pubic symphysis, and was drawn parallel to the margin of the X-ray. PT was defined as and determined by measuring the angle between these two lines. 


\section{Statistical analysis}

Statistical analysis was performed using SPSS 23.0 for Mac (IBM Corporation, Armonk NY). Two-way mixed effects model intraclass correlation coefficients were obtained between the two observers measuring the APP for both pre-BHR measurements and post-BHR measurements to ascertain the level of agreement regarding the measurements. Next, Pearson correlation coefficients were obtained between the pre-BHR measurements and postBHR measurements for both observers to assess whether the APP was altered and to what degree. Mean pre-BHR and post-BHR measurements for both observers were also obtained. The absolute value of the difference between pre-BHR and post-BHR was calculated for both observers $[\triangle \mathrm{APP}=$ abs $($ pre-BHR APP - post BHR APP) $]$. All tests

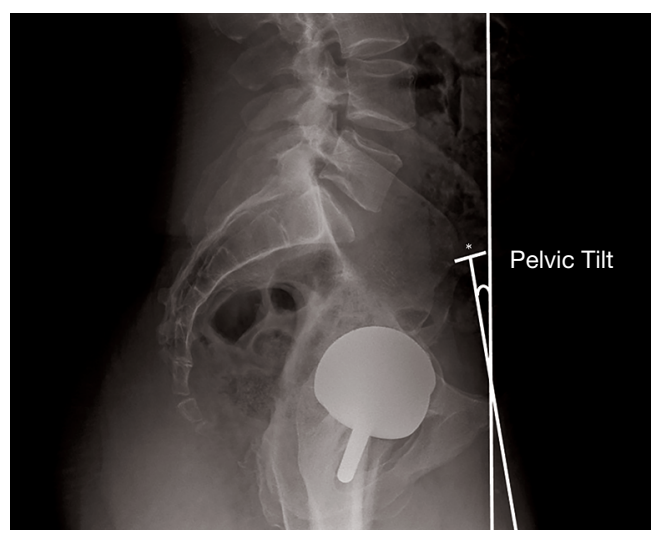

Figure 1 Measurement of pelvic tilt, with respect to the anterior pelvic plane. *, midpoint of the Anterior Superior Iliac Spine. were two-sided and to a significance of $\mathrm{P}<0.05$.

Future studies will compare these differences in PT to changes found following THA.

\section{Results}

The mean PT for observer A preoperatively was $0.7^{\circ}$ [standard deviation (SD) $\pm 6.6^{\circ}$ ], compared to a mean of $0.4^{\circ}\left(\mathrm{SD} \pm 6.1^{\circ}\right)$ for observer B. Mean post-operative PT for observer A was $-1.2^{\circ}\left(\mathrm{SD} \pm 6.2^{\circ}\right)$, while for observer $\mathrm{B}$ it was $-1.2^{\circ}\left(\mathrm{SD} \pm 6.0^{\circ}\right)$.

Strong correlations between pre-operative and postoperative PT measurements were observed for both observer A $(\mathrm{R}=0.829 ; \mathrm{P}<0.001)$ and observer $\mathrm{B}(\mathrm{R}=0.837 ; \mathrm{P}<0.001)$. Categorical analysis of the difference in pre-operative and post-operative PT measurements for observer A revealed that $82.5 \%$ of patients had a variation $<5^{\circ}, 15.8 \%$ had a variation between $5-10^{\circ}$, and $1.8 \%$ had a variation greater than $10^{\circ}$. Similarly, the difference in pre-operative and postoperative PT measurements for observer B revealed that $80.6 \%$ of patients had a variation $<5^{\circ}, 17.8 \%$ had a variation between $5-10^{\circ}$, and $1.6 \%$ had a variation $>10^{\circ}$.

The level of agreement between both observers was strong for pre-operative [intraclass correlation coefficient $(\mathrm{R})=0.987 ; 95 \%$ confidence interval $(\mathrm{CI}), 0.963-0.981$; $\mathrm{P}<0.001$ ] and post-operative (intraclass $\mathrm{R}=0.985 ; 95 \% \mathrm{CI}$, 0.963-0.981; $\mathrm{P}<0.001) \mathrm{PT}$ measurements.

\section{Conclusions}

In the present study, PT was determined before and 1 year after hip resurfacing, using standing lateral radiographs,

Table 1 Reported changes in pelvic tilt following total hip arthroplasty

\begin{tabular}{|c|c|c|c|}
\hline Study & $\begin{array}{l}\text { Sample } \\
\text { size }\end{array}$ & $\begin{array}{l}\text { Post-operative } \\
\text { imaging }\end{array}$ & Differences in pre-op and post-op pelvic tilt \\
\hline Murphy et al., 2013 & 30 & 1-year & $\begin{array}{l}\left.\text { Supine: } 1.9^{\circ} \pm 3.3^{\circ} \text { (range, }-8.5^{\circ} \text { to } 5.5^{\circ}\right), P=0.004 \text {; standing: } 0.5^{\circ} \pm 3^{\circ} \text { (range, }-5^{\circ} \text { to } \\
\left.7.15^{\circ}\right) ; P=0.34\end{array}$ \\
\hline Blondel et al., 2009 & 50 & 3-year & Standing: $3^{\circ} \pm 0.3^{\circ}\left(\right.$ range,$-5^{\circ}$ to $\left.14^{\circ}\right), P>0.05$ \\
\hline Taki et al., 2012 & 86 & $\begin{array}{l}\text { 1-year, 2-year, } \\
\text { 3-year, and } \\
4 \text {-year }\end{array}$ & $\begin{array}{l}\text { Supine: } 1 \text {-year }=2.7^{\circ} \pm 4.4^{\circ}(P<0.05), 2 \text {-year }=2.6^{\circ} \pm 3.9^{\circ}(P<0.05), 3 \text {-year }=3.0^{\circ} \pm 4.2^{\circ} \\
(P<0.05), 4 \text {-year }=2.6^{\circ} \pm 3.2^{\circ}(P<0.05) \text {; Standing: 1-year }=3.9^{\circ} \pm 6.2^{\circ}(P<0.05), 2 \text {-year } \\
=5.3^{\circ} \pm 7.1^{\circ}(P<0.05), 3 \text {-year }=5.1^{\circ} \pm 5.7^{\circ}(P<0.05), 4 \text {-year }=5.2^{\circ} \pm 6.2^{\circ}(P<0.05)\end{array}$ \\
\hline Maratt et al., 2016 & 138 & 6-weeks & $-0.3^{\circ} \pm 3.6^{\circ}$ (range: $-9.6^{\circ}$ to $\left.13.5^{\circ}\right) ; P=0.395$ \\
\hline Nishiwaki et al., 2018 & 335 & 1 day & $0.5^{\circ} \pm 3.5^{\circ}$ (range: $-12.9^{\circ}$ to $21.7^{\circ}$ ); $\mathrm{P}=0.395$ \\
\hline Ishida et al., 2011 & 149 & 1-year & $-8.2 \pm 7.0,-2.5 \pm 5.9,-1.5 \pm 6.9$, and $2.2 \pm 6.7 ; P<0.05$ \\
\hline
\end{tabular}


measuring the angle between the APP, represented by a line connecting the ASIS to pubic symphysis, and the vertical plane $(17,20)$. This method is simple, cost-effective, can be incorporated into computer-navigation systems, and approximates acetabular orientation during walking, where components are subject to greatest load-bearing and wear $(2,17,20)$. However, one disadvantage of this method is that it is dependent on observer accuracy. Hence the purpose of this study was to investigate how native PT changes after hip resurfacing, and also to investigate whether determination of the APP angle via lateral standing radiographs is a reproducible method for evaluating $\mathrm{PT}$ in both native and resurfaced hips. The present results suggest that for a majority of patients, PT is minimally affected by hip resurfacing, with average changes being less than $1^{\circ}$. In over $80 \%$ of patients, the angle of the APP on standing lateral radiographs changed by less than 5 degrees in 1-year after surgery. Furthermore, less than $2 \%$ of patients showed a change of greater than 10 degrees 1 -year post-surgery. Pre- and postoperative measurements also showed high correlation with coefficients above 0.82 for both observers. Therefore, hip resurfacing seems to have very minor impact on PT which can be assessed using our cost-effective methodology with high degree of inter-observer reliability.

Several analyses of changes in PT after THA have found similarly minimal difference post-surgery. Also using standing lateral radiographs of 50 patients, Blondel et al. found no significant difference in PT, before, and three years after THA, with $95 \%$ of patients showing less than 5 degrees of variation (17). Pinot et al similarly found no significant variation in $\mathrm{PT}$ in 19 patients before and after THA on standing lateral radiographs (30). DiGioia et al. also found no significant variation in PT 3-months post THA on standing lateral radiographs (20). More recently Maratt et al. found no significant change in PT on standing lateral radiographs in 138 patients 6 weeks after THA (26). Murphy et al. also investigated changes in PT after THA in 30 patients using $\mathrm{CT}$ and $\mathrm{AP}$ radiographs, and found changes of less than 1 degree on average 1 year after surgery (4). These findings suggest that preoperative measurements of PT can be used to guide placement of the acetabular component to improve its postoperative functional orientation.

Some studies, however, have found that PT is altered after THA, usually trending posteriorly, or that postoperative changes vary widely between patients $(8,10-13)$. It is purported this could confound the accuracy of computer navigation systems that account for preoperative PT, as demonstrated by Paratte et al. (18). It is possible other patient specific factors should be considered during preoperative planning, particularly those which are associated with greater postoperative changes in PT. Ishida et al found that greater changes are associated with coxalgia due to acetabular dysplasia, or severe anterior PT due to contracture of the hip joint (29). Kyo et al. and Tamura et al. both found that spinal factors such as preoperative compression fractures, spondylolisthesis, and disk-space narrowing are predictive of greater postoperative changes in PT $(28,31)$. Suzuki et al. found that posterior PT tends to increase over time in older patients with smaller lumbo-lordotic angle, and greater preoperative changes in PT between standing and sitting position (10). Taki et al. also demonstrated that postoperative changes in PT tend to be greater in older patients (25). Recently Nishiwaki et al. found that postoperative changes in PT were more variable in patients who had a wider contralateral joint space (27).

It is uncertain however how relevant these potential confounding factors are to the hip resurfacing population, as many are associated with older age, and degenerative conditions. Regardless, it is reasonable to conclude that patient-specific PT should be considered as an important piece of perhaps more comprehensive preoperative planning for both THA and resurfacing. Several studies have demonstrated that tilt-adjusted computer navigation guidance may improve outcomes after THA. Babisch et al. used hip navigation planning software to develop a nomogram that adjusted guidance of cup placement for preoperative PT. The nomogram was used for computer-navigated THA in 98 patients. The authors reported no dislocations at 1 year follow up, with $99 \%$ of cup anteversion values, and $97 \%$ of cub abduction values within the targeted range (15). Inaba et al. demonstrated similarly favorable results in 75 patients who had undergone THA using CT-based navigation, and preoperative planning with consideration of PT (24).

There are several methods for evaluating PT, reported on in the literature. However, many rely on CT, which is expensive and involves high doses of ionizing radiation, or involve complex, time-consuming calculations based on AP radiographs, which that may be subject to inaccuracy $(4,21,24,25,32,33)$. The present study measured PT on lateral radiographs of the pelvis, with the patient in standing position, as described by DiGoia et al. (20), and reported on in a number of other studies $(14,15,17,26,29)$. An observer draws two lines, one line representing the APP, connecting the ASIS and pubic symphysis, and a second line representing the vertical plans, intersecting the first line at the ASIS, and parallel to the margin of the X-ray. PT is 
approximated by the angle between these two lines. This method is cost-effective, can be performed within minutes, and because it measures the angle of the APP, can be easily incorporated into a majority of existing CT navigation systems, which rely on reconstruction of the APP to guide placement of components.

Furthermore, in resurfacing it is arguably most important to optimize orientation of components in standing position, because this provides the closest approximation to walking, where implants are subject to greatest load bearing and wear $(2,17,20)$. Consideration of sitting PT, and changes in PT moving from sitting to standing may also be important, as posterior dislocation often occurs when rising from sitting position. However, very low rates of dislocation post resurfacing, suggest that sitting PT may be more relevant to THA (34).

One drawback of this method is that it is not automated, and rather is dependent on the accuracy of the observer. However, our results demonstrated high accuracy between observers, with intraclass correlation coefficients of 0.987 for preoperative measurements, and 0.985 for postoperative measurements. Correlation coefficients between pre- and postoperative measurements were also similar between observers at 0.829 for observer $\mathrm{A}$, and 0.837 for observer B. Blondel et al. similarly found these measurements are reliable and reproducible between multiple observers (17).

This paper is not without limitations. Surgeries were performed by one surgeon in an academic setting and could be strengthened by including additional surgeons in different settings. All of the patients in this study were men and a vast majority of the patients were white $(82.9 \%)$, primarily limiting our conclusions to those demographics. Females were also excluded from this study since hip resurfacing using the BHR device is now indicated only in males. Also, a substantial portion (43.4\%) of the patients lacked measurements for observer B, which limits the reliability of intraclass correlation coefficients as well as the correlation coefficients for that observer. However, measurements were still completed by both observers in over 125 patients, a larger sample than many other similar studies. Additionally, categorical analyses revealed minimal deviations between observer measurements, and this method of measuring PT has been previously demonstrated to be reproducible (17).

In conclusion, this unique study specifically investigated changes in PT in hip resurfacing patients with emphasizing high level of inter-observer reliability. We found that following hip resurfacing, changes in PT, on average, were less than $1^{\circ}$ and had a relatively narrow range. This is contradictory to studies on the effects of THA on PT, which have yielded larger changes with wider variability. Future studies are needed to determine optimal orientation of the acetabular component in resurfacing, and clinical outcomes when preoperative PT is incorporated into surgical planning. Nevertheless, this novel study will serve to expand our knowledge on understanding dynamic changes in PT particularly in the specific patient population of hip resurfacing.

\section{Acknowledgments}

None.

\section{Footnote}

Conflicts of Interest: MA Mont reports the following: AAOS: Board or committee member; American Association of Hip and Knee Surgeons: Board or committee member; Cymedica: Paid consultant; DJ Orthopaedics: Paid consultant; Research support; Flexion Therapeutics: Paid consultant; Johnson \& Johnson: Paid consultant; Research support; Journal of Arthroplasty: Editorial or governing board; Journal of Knee Surgery: Editorial or governing board; Knee Society: Board or committee member; Medicus Works LLC: Publishing royalties, financial or material support; Microport: IP royalties; National Institutes of Health (NIAMS \& NICHD): Research support; Ongoing Care Solutions: Paid consultant; Research support Orthopedics: Editorial or governing board; Orthosensor: Paid consultant; Research support; Pacira: Paid consultant; Peerwell: Paid consultant; Stock or stock Options; Performance Dynamics: Paid consultant; Pfizer: Paid consultant; Skye Biologics: Paid consultant; Stryker: IP royalties; Paid consultant; Research support; Surgical Techniques International: Editorial or governing board; Tissue Gene: Paid consultant; TissueGene: Research support; Up-to Date: Publishing royalties, financial or material support; USMI: Stock or stock Options; Wolters Kluwer Health - Lippincott Williams \& Wilkins: Publishing royalties, financial or material support. PJ Brooks reports the following: Smith \& Nephew: Paid consultant; Zimmer: Paid consultant. The other authors have nothing to disclose.

Ethical Statement: IRB approval (\#17-393) was obtained for this study. 


\section{References}

1. Girard J. Hip Resurfacing: International Perspectives: Review Article. HSS J 2017;13:7-11.

2. Eilander W, Harris SJ, Henkus HE, et al. Functional acetabular component position with supine total hip replacement. Bone Joint J 2013;95-B:1326-31.

3. Grammatopoulos G, Pandit HG, da Assunção R, et al. The relationship between operative and radiographic acetabular component orientation: which factors influence resultant cup orientation? Bone Joint J 2014;96-B:1290-7.

4. Murphy WS, Klingenstein G, Murphy SB, et al. Pelvic tilt is minimally changed by total hip arthroplasty. Clin Orthop Relat Res 2013;471:417-21.

5. Romanowski JR, Swank ML. Imageless navigation in hip resurfacing: avoiding component malposition during the surgeon learning curve. J Bone Joint Surg Am 2008;90 Suppl 3:65-70.

6. Langston J, Pierrepont J, Gu Y, et al. Risk factors for increased sagittal pelvic motion causing unfavourable orientation of the acetabular component in patients undergoing total hip arthroplasty. Bone Joint J 2018;100B:845-52.

7. Sultan AA, Khlopas A, Piuzzi NS, Chughtai M, Sodhi N, Mont MA. The Impact of Spino-Pelvic Alignment on Total Hip Arthroplasty Outcomes: A Critical Analysis of Current Evidence. J Arthroplasty 2018;33:1606-16.

8. Sultan AA, Khlopas A, Udo-Inyang I, et al. Hip Osteoarthritis Patients Demonstrated Marked Dynamic Changes and Variability in Pelvic Tilt, Obliquity, and Rotation: A Comparative, Gait-Analysis Study. Surg Technol Int 2018;32:285-92.

9. Harwin SF, Sodhi N, Ehiorobo J, et al. Outcomes of Dual Mobility Acetabular Cups in Total Hip Arthroplasty Patients. Surg Technol Int 2019;34:367-70.

10. Suzuki H, Inaba Y, Kobayashi N, et al. Postural and Chronological Change in Pelvic Tilt Five Years After Total Hip Arthroplasty in Patients With Developmental Dysplasia of the Hip: A Three-Dimensional Analysis. J Arthroplasty 2016;31:317-22.

11. Rousseau MA, Lazennec JY, Boyer P, et al. Optimization of total hip arthroplasty implantation: is the anterior pelvic plane concept valid? J Arthroplasty 2009;24:22-6.

12. Sultan AA, Cantrell WA, Khlopas A, et al. Evidence-Based Management of Trunnionosis in Metal-on-Polyethylene Total Hip Arthroplasty: A Systematic Review. J Arthroplasty 2018;33:3343-53.

13. Cleveland Clinic Orthopaedic Arthroplasty. The
Association Between Readmission and Patient Experience in a Total Hip Arthroplasty Population. J Arthroplasty 2018;33:1668-74.

14. Pierrepont J, Hawdon G, Miles BP, et al. Variation in functional pelvic tilt in patients undergoing total hip arthroplasty. Bone Joint J 2017;99-B:184-91.

15. Babisch JW, Layher F, Amiot LP. The rationale for tiltadjusted acetabular cup navigation. J Bone Joint Surg Am 2008;90:357-65.

16. Lazennec JY, Thauront F, Robbins CB, et al. Acetabular and Femoral Anteversions in Standing Position are Outside the Proposed Safe Zone After Total Hip Arthroplasty. J Arthroplasty 2017;32:3550-6.

17. Blondel B, Parratte S, Tropiano P, et al. Pelvic tilt measurement before and after total hip arthroplasty. Orthop Traumatol Surg Res 2009;95:568-72.

18. Parratte S, Pagnano MW, Coleman-Wood K, et al. The 2008 frank stinchfield award: Variation in postoperative pelvic tilt may confound the accuracy of hip navigation systems. Clin Orthop Relat Res 2009;467:43-9.

19. Barbier O, Skalli W, Mainard L, Mainard D. The reliability of the anterior pelvic plane for computer navigated acetabular component placement during total hip arthroplasty: Prospective study with the EOS imaging system. Orthop Traumatol Surg Res 2014;100:S287-91.

20. DiGioia AM, Hafez MA, Jaramaz B, et al. Functional pelvic orientation measured from lateral standing and sitting radiographs. Clin Orthop Relat Res 2006;453:272-6.

21. Zahn RK, Grotjohann S, Ramm H, et al. Pelvic tilt compensates for increased acetabular anteversion. Int Orthop 2016;40:1571-5.

22. Lewis CL, Laudicina NM, Khuu A, et al. The Human Pelvis: Variation in Structure and Function During Gait. Anat Rec (Hoboken) 2017;300:633-42.

23. Zahn RK, Grotjohann S, Pumberger M, et al. Influence of pelvic tilt on functional acetabular orientation. Technol Health Care 2017;25:557-65.

24. Inaba Y, Kobayashi N, Suzuki H, et al. Preoperative planning for implant placement with consideration of pelvic tilt in total hip arthroplasty: postoperative efficacy evaluation. BMC Musculoskelet Disord 2016;17:280.

25. Taki N, Mitsugi N, Mochida Y, et al. Change in pelvic tilt angle 2 to 4 years after total hip arthroplasty. J Arthroplasty 2012;27:940-4.

26. Maratt JD, Esposito CI, McLawhorn AS, et al. Pelvic Tilt in Patients Undergoing Total Hip Arthroplasty: When Does it Matter? J Arthroplasty 2015;30:387-91.

27. Nishiwaki T, Hata R, Oya A, et al. Pelvic Tilt 
Displacement Before and After Artificial Hip Joint Replacement Surgery. J Arthroplasty 2018;33:925-30.

28. Kyo T, Nakahara I, Miki H. Factors Predicting Change in Pelvic Posterior Tilt After THA. Orthopedics 2013;36:e753-9.

29. Ishida T, Inaba Y, Kobayashi N, et al. Changes in pelvic tilt following total hip arthroplasty. J Orthop Sci 2011;16:682-8.

30. Pinoit $\mathrm{Y}$, May $\mathrm{O}$, Girard J, et al. Low accuracy of the anterior pelvic plane to guide the position of the cup with imageless computer assistance: variation of position in 106 patients. Rev Chir orthopédique réparatrice l'appareil Mot. 2007;93:455-60.

Cite this article as: Samuel LT, Warren J, Rabin JM, Acuña AJ, Shuster A, Patterson J, Mont MA, Brooks PJ. Perioperative changes in pelvic tilt after hip resurfacing using a direct lateral approach. Ann Transl Med 2019. doi: 10.21037/atm.2019.05.67
31. Tamura S, Takao M, Sakai T, et al. Spinal factors influencing change in pelvic sagittal inclination from supine position to standing position in patients before total hip arthroplasty. J Arthroplasty 2014;29:2294-7.

32. Tannast M, Murphy SB, Langlotz F, et al. Estimation of pelvic tilt on anteroposterior X-rays--a comparison of six parameters. Skeletal Radiol 2006;35:149-55.

33. Yun H, Murphy WS, Ward DM, et al. Effect of Pelvic Tilt and Rotation on Cup Orientation in Both Supine and Standing Positions. J Arthroplasty. 2018;33:1442-8.

34. Brooks PJ. Hip resurfacing: a large, US single-surgeon series. Bone Joint J 2016;98-B:10-3. 